\title{
Enfermedad por bacilo de Calmette-Guérin (BCG) y deficiencia del receptor $\beta-1$ de interleuquina 12. Experiencia clínica de dos casos en una familia y un caso aislado
}

\author{
Alexis Strickler, Amir Pérez, Migdy Risco y Silvanna Gallo
}

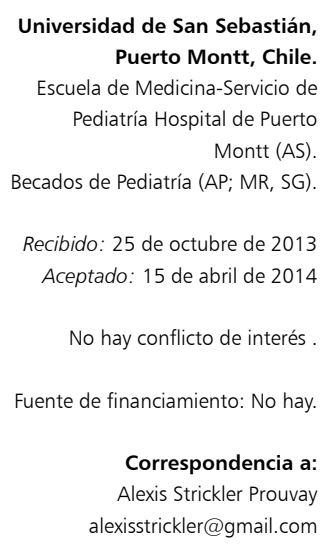

alexisstrickler@gmail.com

\author{
Bacillus Calmette-Guérin (BCG) disease and interleukin 12 receptor $\beta 1$ deficiency: \\ Clinical experience of two familial and one sporadic case
}

BCG disease has been reported in primary and secondary immunodeficiency and as Mendelian Susceptibility to Mycobacterial Diseases (MSMD). Investigation of this syndrome has led to the identifications of a series of genetic, inherited defects in the IL-12/IFN- $\gamma$ axis. MSMD-causing mutations have been found in seven autosomal and two X-linked genes. In these patients, local or disseminated vaccine BCG infections are common. We report a clinical series including two infants with left axillary adenitis ipsilateral to the site of neonatal BCG immunization; one of them member of a family with two previously reported cases and a single sporadic case. All of them were diagnosed sequentially in Puerto Montt, Chile. The aim of this report is to notify the first Chilean disseminated BCG patients without previous immunodeficiency, in whom it was possible to identify an underlying immunodeficiency, although specific tests for IL-12/IFN- $\gamma$ axis was no performed in our country. Clinical suspicion and international collaboration permitted to confirm IL12-R $\beta 1$ deficiency in 2 of 3 familial cases and a sporadic case.

Key words: Primary immunodeficiency, BCG, Mendelian susceptibility to mycobacterial diseases, IL-12/IFN- $\gamma$ axis, IL-12R $\beta 1$ deficiency.

Palabras clave: Inmunodeficiencia primaria, BCG, susceptibilidad mendeliana a infecciones micobacterianas, eje IL-12/ IFN- $\gamma$, deficiencia del receptor $\beta 1$ de IL12.

\section{Introducción}

L a vacuna BCG, que contiene una cepa del bacilo Mycobacterium bovis vivo, atenuado, es administrada rutinariamente a todos los recién nacidos poco después del parto, en los países donde existen aún altas tasas de TBC. Su eficacia ha sido documentada en prevenir las formas graves, potencialmente letales, de tuberculosis (TBC), como enfermedad diseminada (TBC miliar) y enfermedad meníngea ${ }^{1-8}$. Sin embargo, esta vacunación puede producir reacciones adversas, de diversa magnitud, en niños con inmunodeficiencias primarias (IDP) y secundarias previamente diagnosticadas, aunque también en un reducido grupo de niños aparentemente sanos, en los cuales los exámenes inmunológicos de rutina son normales, pero que padecen de una mutación genética. Se trata de un defecto heredado en una molécula del sistema inmunológico, conocida como síndrome de susceptibilidad mendeliana a enfermedades micobacterianas SMEM (en inglés Mendelian susceptibility to mycobacterial disease-MSMD) $)^{9-16}$. La investigación de este síndrome en los últimos 17 años (1996-2013), ha llevado a la identificación de defectos en el eje IL-12/ IFN- $\gamma$, habiéndose identificado hasta hoy mutaciones en siete genes autosómicos y dos ligados al cromosoma X. Adicionalmente, la heterogeneidad alélica ha contribuido a definir hasta 17 alteraciones genéticas, con defectos parciales y completos, de herencia dominante y recesiva, en las moléculas involucradas en el circuito IL-12/IFN- $\gamma$, ya sea en las respuestas celulares a interferón- $\gamma$ o en la inducción de IFN- $\gamma$ dependiente de IL12 (Figura 1). Una de ellas es la deficiencia del receptor $\beta$ - 1 de IL12 $(\mathrm{IL} 12 \mathrm{R} \beta 1)^{17-31}$. Aunque en la clasificación más reciente de IDP, SMEM constituye un grupo específico de IDP ${ }^{25}$, este síndrome puede presentarse en otras IDP en las que se compromete la respuesta inmune (RI) celular tipo $\mathrm{TH} 1$, tales como inmunodeficiencia combinada severa, enfermedad granulomatosa crónica, síndrome de hiper IgE, síndrome de hiper IgM e inmunodeficiencia secundaria a infección por VIH o tratamientos inmunosupresores ${ }^{15-23,32,33}$.

La deficiencia de IL-12 R $\beta 1$ fue descrita por primera vez en 1998, y es la alteración genética más común en el eje IL-12/IFN- $\gamma$. Ha sido diagnosticada en más de 40 países en los cinco continentes ${ }^{21,27-29,34-37}$. Entre los pacientes reportados por los investigadores del Laboratorio de Genética Humana de Enfermedades Infecciosas, Facultad de Medicina Necker, París, Francia, formando parte de un 


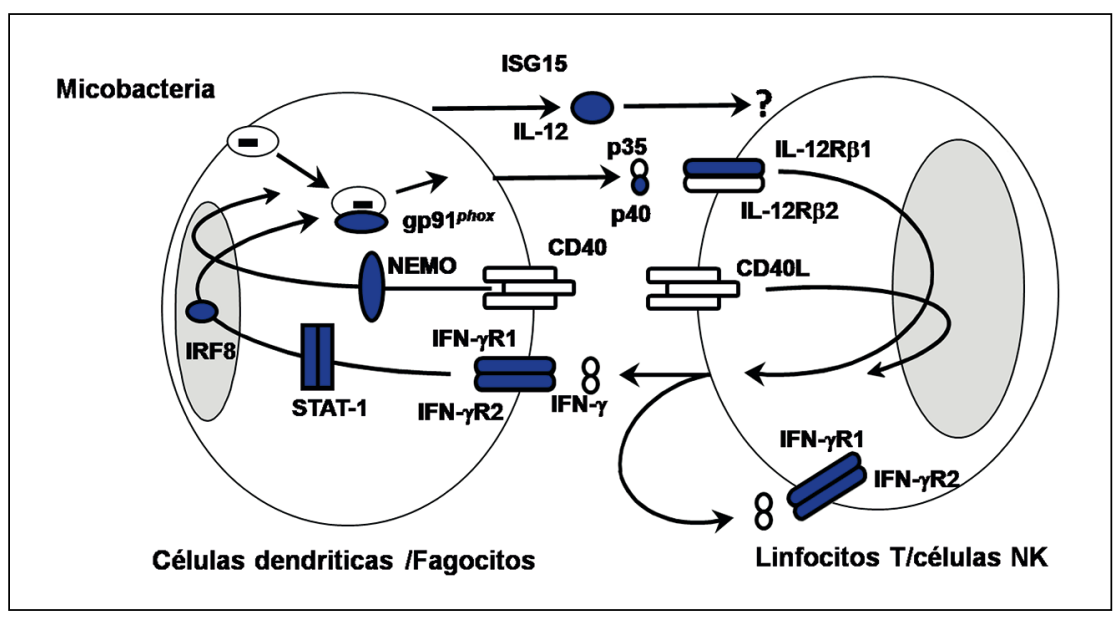

Figura 1. Predisposición genética a infecciones por micobacterias (defecto del eje IL-12-IFN- $\gamma$ ). Representación esquemática de las células que intervienen en la respuesta inmunitaria dirigida contra micobacterias. Las células presentadoras de antígeno (fagocitos y células dendríticas) infectadas secretan IL-12, citoquina heterodimérica (compuesta de dos sub-unidades p35 y p40). La IL-12 va a fijarse a su receptor (compuesto de dos sub-unidades $\beta 1$ y $\beta 2$ ) en la superficie de células NK y de linfocitos T y va a inducir la producción de IFN- $\gamma$. EI IFN- $\gamma$ se fijará sobre su receptor (compuesto de dos cadenas IFN- $\gamma$ R1 y IFN- $\gamma$ R2) y va a permitir a STAT1 fosforilarse y translocarse hacia el núcleo para inducir la producción de citoquinas como IL-12. La proteína NEMO tiene una función reguladora en la vía NF-KB e interviene en la producción de IL-12 a través de las moléculas CD40-CD40L. En las moléculas representadas en azul, se ha identificado mutaciones en pacientes con SMEM (Fuente: Jacinta Bustamante, Laboratorio de Genética Humana de Enfermedades Infecciosas Necker, Institut Imagine-París, Francia). estudio colaborativo, se encuentran varios niños chilenos, incluyendo los casos incluidos en este artículo. Para el estudio genético y la difusión de datos contenidos en las historias clínicas, se obtuvo el consentimiento informado de los padres y del Director del Hospital Puerto Montt. Objetivos de este reporte son dar a conocer a la comunidad científica nacional, algunos de los casos diagnosticados en nuestro país, en los que se logró demostrar la deficiencia del receptor $\beta 1$ de IL12, gracias a la interrelación con centros mundiales de investigación genética y, adicionalmente, contribuir a la sospecha clínica, estudio y tratamiento precoz de la enfermedad por BCG, lo que no sólo mejorará el pronóstico individual, sino que contribuirá a aportar casos a los centros de investigación que permita aumentar el conocimiento de estas patologías.

\section{Casos clínicos}

Los casos señalados en este trabajo como 1 y 2 fueron previamente reportados como enfermedad BCG, correspondiendo a los casos 4 y 5 de esa publicación ${ }^{18}$. El caso 2 (caso 5 en referencia 18 ) continúa en estudio ya que no se identificó la alteración biomolecular y genética subyacente $^{18}$. Los casos 3 y 4 que integran este reporte, fueron diagnosticados y tratados con posterioridad. En todos los casos, y en relación al diagnóstico de los dos últimos, se envió muestra de los pacientes, hermanos, y padres para estudio del eje IFN- $\gamma /$ IL12, al Laboratorio de Genética Humana de Enfermedades Infecciosas- Facultad de Medicina Necker, París, Francia, identificándose deficiencia del receptor $\beta 1$ de IL12, en los casos 1, 3 y 4 (Tabla 1).

A continuación se detallan los casos nuevos.

Caso 3. Paciente femenino, residente en Aulen, Hualaihue, Palena-Región de Los Lagos. RNT 37 semanas, peso y talla de nacimiento: $2.800 \mathrm{~g} / 48 \mathrm{~cm}$. Recibió vacuna BCG al nacer. Antecedentes patológicos: candidiasis oral leve y bronquiolitis por VRS al mes de vida. Sin antecedentes de contacto TBC. Edad actual: cuatro años 10 meses. Hermano dos años mayor y padres adultos jóvenes sanos, sin consanguinidad. La paciente es prima hermana por padre y madre del caso número 1 y prima en segundo grado por parte de padre del caso 2 (Figura 2).

A los tres meses de edad, presentó adenomegalia axilar

\begin{tabular}{|c|c|c|c|c|}
\hline Caso & Mutación & Gen & Tipo de defecto & Trabajo científico (Revista o Prensa) \\
\hline Caso 1 & pC186X o c558C3A & Homocigoto & Defecto completo IL12Rß1 & $\begin{array}{l}\text { IL12-R } \beta 1 \text { Deficiency: Mutation update and description of IL12-R } \beta 1 \text { variation database. } \\
\text { Human Mutation 2013; } 34 \text { (10); 1329-39 (37) / Ref } 36 \\
\text { Ref (18) }\end{array}$ \\
\hline Caso 2 & No encontrado & & & $\operatorname{Ref}(18)$ \\
\hline Caso 3 & pC186X o c558C3A & Homocigoto & Defecto completo IL12Rß1 & $\begin{array}{l}\text { IL12-Rß1 Deficiency: Mutation update and description of IL12-R } \beta 1 \text { variation database. } \\
\text { Human Mutation 2013; } 34 \text { (10); 1329-39 (37) Ref } 36\end{array}$ \\
\hline Caso 4 & g169del o S57VjsX73 & Homocigoto & Defecto completo IL12R $\beta 1$ & Beaucondrey et al. Medicine 2010 (34) Ref 36 \\
\hline
\end{tabular}


Figura 2. Genograma de la familia de tres casos de enfermedad BCG diseminada. Los números romanos corresponden a las generaciones. Los números arábigos corresponden al número de hijos; y los círculos negros a los individuos afectados.

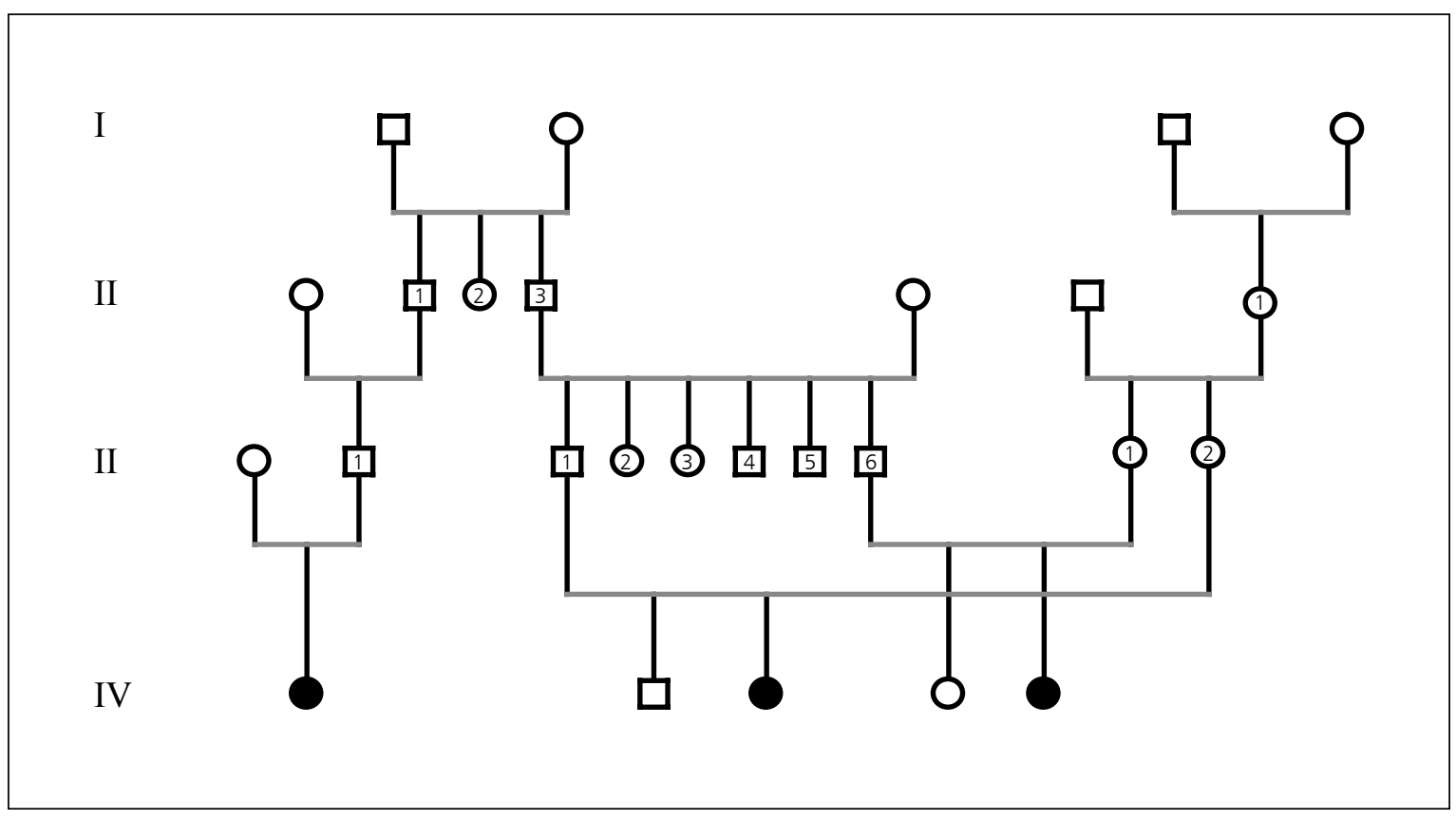

\section{Tabla 2. Microbiología-ubicación-tratamiento y evolución clínica casos de enfermedad BCG}

\begin{tabular}{|c|c|c|c|c|c|c|}
\hline Caso & Cultivo & $\begin{array}{l}\text { Susceptibilidad } \\
\text { in vitro }\end{array}$ & Infección ubicación & Tratamiento de la infección BCG* & $\begin{array}{l}\text { Duración del } \\
\text { tratamiento }\end{array}$ & Evolución y seguimiento \\
\hline 1 & No disponible & & $\begin{array}{l}\text { Adenitis axilar izquierda BCG, } \\
\text { candidiasis oral y glúteo- } \\
\text { genital }\end{array}$ & $\begin{array}{l}\text { HIN, RFP, Z }(5,10,25) \mathrm{mg} / \mathrm{kg} / \mathrm{día} \\
\text { HIN, RFP }(15,10) \mathrm{mg} / \mathrm{kg} / \mathrm{bisemanal} \\
5 \text { meses sin terapia. } \\
\text { HIN, RFP, Z }(5,10,25) \mathrm{mg} / \mathrm{kg} / \mathrm{día} \\
\text { HIN, RFP }(15,10) \mathrm{mg} / \mathrm{kg} / \mathrm{dia}\end{array}$ & $\begin{array}{l}2 \text { meses } \\
4 \text { meses } \\
2 \text { meses } \\
6 \text { meses }\end{array}$ & $\begin{array}{l}\text { Evolución clínica favorable } \\
\text { Último control año } 2012\end{array}$ \\
\hline 2 & $\begin{array}{l}\text { Mycobacterium bovis } \\
\text { subespecie BCG }\end{array}$ & Sensible & $\begin{array}{l}\text { Adenitis axilar y supraclavicu- } \\
\text { lar izquierda BCG, linfonodos } \\
\text { mesentéricos. }\end{array}$ & $\begin{array}{l}\text { HIN, RFP }(5,10) \mathrm{mg} / \mathrm{kg} / \mathrm{día} \\
\text { HIN, RFP, Z, ETA }(15,20,20,20) \mathrm{mg} / \mathrm{kg} / \mathrm{día} \\
24 \text { días sin tratamiento } \\
\text { HIN, RFP, Z, ETA }(15,20,20,20) \mathrm{mg} / \mathrm{kg} / \mathrm{día} \\
\text { HIN, RFP, ETA }(15,20,20) \mathrm{mg} / \mathrm{kg} / \mathrm{dia} \\
\text { HIN, RFP, ETA }(15,20,20) \mathrm{mg} / \mathrm{kg} / \mathrm{bisemanal}\end{array}$ & $\begin{array}{l}1 \text { mes } \\
1 \text { mes } \\
4 \text { meses } \\
5 \text { meses } \\
1 \text { mes }\end{array}$ & $\begin{array}{l}\text { Evolución clínica favorable } \\
\text { Último control año } 2012\end{array}$ \\
\hline 3 & $\begin{array}{l}\text { Mycobacterium bovis } \\
\text { subespecie } B C G\end{array}$ & $\begin{array}{l}\text { Resistente a } \\
\text { pirazinamida }\end{array}$ & Adenitis axilar izquierda BCG & HIN, RFP, ETA $(15,20,20) \mathrm{mg} / \mathrm{kg} / \mathrm{dia}$ & 18 meses & $\begin{array}{l}\text { Evolución clínica favorable } \\
\text { Último control año } 2012\end{array}$ \\
\hline 4 & $\begin{array}{l}\text { Mycobacterium bovis } \\
\text { subespecie } B C G\end{array}$ & $\begin{array}{c}\text { No se logró } \\
\text { evaluar }\end{array}$ & $\begin{array}{l}\text { Adenitis axilar izquierda BCG, } \\
\text { candidiasis oral, } \\
\text { dermatosis purpúrica pig- } \\
\text { mentada }\end{array}$ & $\begin{array}{l}\text { HIN, RFP, ETA }(5,5,10) \mathrm{mg} / \mathrm{kg} / \mathrm{día} \\
\text { HIN, RFP, ETA }(5,10,20) \mathrm{mg} / \mathrm{kg} / \mathrm{día}\end{array}$ & $\begin{array}{l}1 \text { mes } \\
13 \text { meses }\end{array}$ & $\begin{array}{l}\text { Evolución clínica favorable } \\
\text { Último control año } 2012\end{array}$ \\
\hline
\end{tabular}

* $\mathrm{HIN}=$ isoniazida $\mathrm{RFP}=$ rifampicina $\mathrm{Z}=$ pirazinamida $\mathrm{ETA}=$ etambutol. 
izquierda de 4 x $4 \mathrm{~cm}$, fistulizada y con tejido granulomatoso. Se le realizó curetaje y luego, por recidiva, se efectúa estudio inmunológico habitual (Tabla 2) y se le indicó tratamiento anti-TBC para enfermedad BCG diseminada, según esquema sudafricano-HIN + RFP + ETA- ${ }^{23}$ el que se mantuvo por 18 meses (Tabla 2). Una biopsia fue informada como: "linfoadenitis granulomatosa crónica de tipo tuberculosa; tinción de Ziehl Nielsen $(+)$ ". Cultivo: Mycobacterium bovis BCG resistente a pirazinamida. La respuesta clínica fue satisfactoria, permaneciendo sana a los cuatro años de seguimiento.

Se realizó secuenciamiento del exoma utilizando los ADN de ambas pacientes (caso 1 y caso 3 ). En ambos casos se identificó una mutación al estado homocigoto en el exón 6 del gen IL12RB1, p.C186X, que se confirmó por método Sanger. Al realizar la segregación familiar, los padres de ambas pacientes resultaron ser heterocigotos para la mutación. Se evaluó la expresión de la proteína IL12B1 usando blastos PHA y no se detectó la expresión de dicha proteína.

Caso 4. Paciente masculino, residente en Huito, Calbuco, Llanquihue-Región de Los Lagos. Edad actual: cinco años. Tercer hijo de padres de 40 y 37 años. sanos, sin consanguinidad. Dos hermanos, de 10 y 13 años. RNT 38 semanas, peso y talla de nacimiento: $3.550 \mathrm{~g} / 51 \mathrm{~cm}$. Recibió vacuna BCG al nacer. Presentó una candidiasis oral leve en el período neonatal. Sin antecedentes de contacto TBC.

Al tercer mes de vida presentó adenomegalia axilar izquierda $10 \times 10 \mathrm{~cm}$, que correspondió histológicamente a linfoadenitis granulomatosa crónica BAAR $(+++)$ con abundantes bacilos subcapsulares. Cultivo positivo para M. bovis variedad BCG. Estudio inmunológico corriente normal (Tabla 2). Se trató con similar esquema para enfermedad BCG diseminada que el caso anterior (Tabla 2). A los cinco meses de tratamiento presentó un nuevo episodio de candidiasis oral y a los siete meses una recidiva de la adenopatía axilar que cedió posteriormente sin modificación del plan de tratamiento del cual completó 16 meses. Se envió muestras del paciente y de la madre al Laboratorio de Genética Humana de Enfermedades Infecciosas-Facultad de Medicina Necker-París. Debido a la asociación clínica de infección micobacteriana y candidiasis, se realizó secuenciamiento usando el método Sanger con lo que se identificó una mutación al estado homocigoto en el exón 3 del gen $I L 12 R B 1$, c.169del. La madre presentaba la mutación al estado heterocigoto. $\mathrm{Se}$ evaluó la expresión de la proteína IL12B1 usando blastos PHA y no se detectó la expresión de dicha proteína. Se concluyó deficiencia de IL12R $\beta 1$ (Tabla 1 y Figura 3). Cinco meses después de suspender el tratamiento, aparecieron adenopatías cervicales derechas interpretadas como de etiología bacteriana, que cedieron con el uso

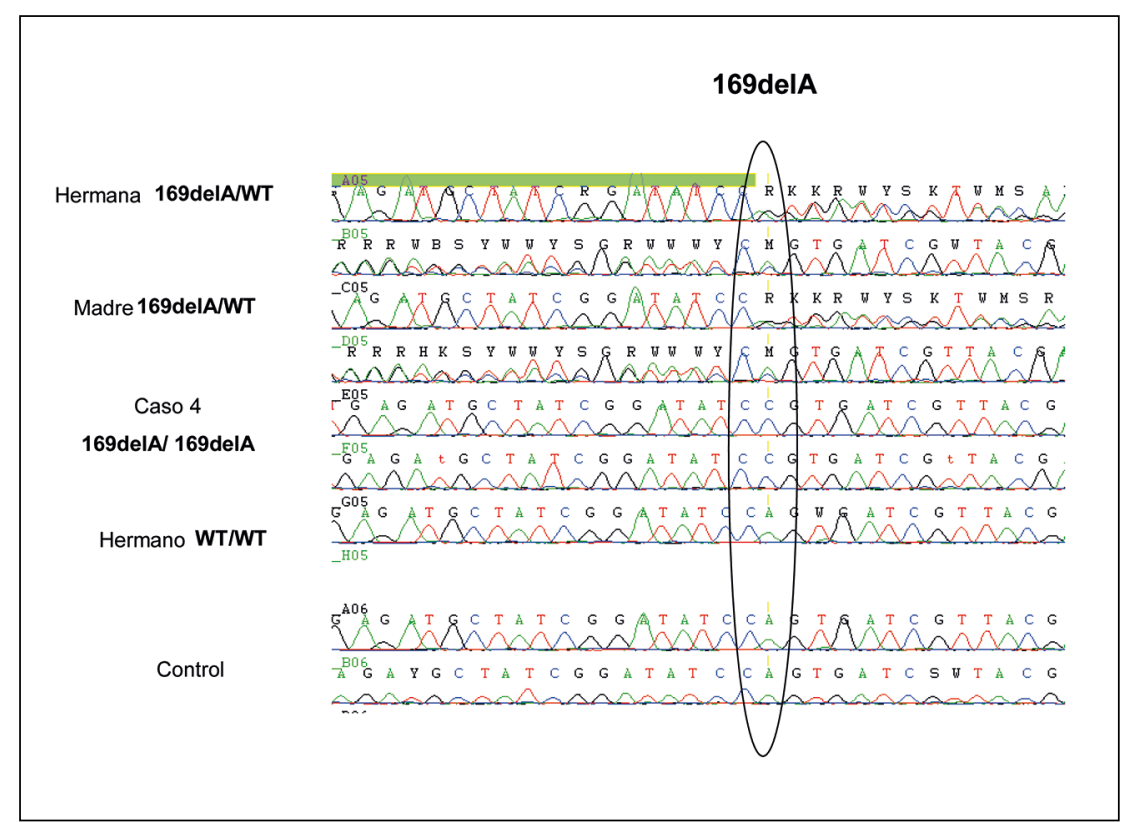

Figura 3. Segregación familiar caso 4, IL12Rß1, c169-delA.

durante dos semanas de cefadroxilo. Siete meses más tarde, (un año después de terminar el tratamiento antimicobacteriano) le aparecieron lesiones purpúricas en las extremidades inferiores, asociadas a trombocitopenia y velocidad de eritrosedimentación (VHS) en aumento. Una biopsia cutánea concluyó: “dermatosis purpúrica pigmentada". El paciente abandonó los controles a los tres años de edad. Un seguimiento telefónico en marzo de 2013 permitió conocer que se encontraba en buenas condiciones generales de salud, sin adenopatías, manteniendo las lesiones cutáneas.

\section{Revisión de literatura científica y discusión}

Los datos obtenidos en animales indican que la RI neonatal $(\mathrm{NN})$ está polarizada hacia RI tipo T colaborador 2 (TH2 en la sigla en inglés). La respuesta tipo colaborador 1 (TH1) se caracteriza por la producción de IFN- $\gamma$, molécula que se requiere para la protección contra micobacterias y virus. La vacuna BCG, única vacuna disponible para la prevención de TBC, induce respuesta TH1 de memoria, similar a la que se observa posteriormente en la vida, por lo que se puede inmunizar al nacer, contrariamente a lo que ocurre con las vacunas polisacáridas contra Streptococcus pneumoniae en que la inmadurez NN afecta la respuesta de los linfocitos $\mathrm{B}$ a antígenos (Ag) independientes de linfocitos T. La respuesta TH1 inducida por BCG es dependiente de las células dendríticas, presentadoras de Ag (CPA), involucradas en el inicio de la RI primaria. Las micobacterias, incluyendo BCG, infectan las células 
dendríticas aumentando su capacidad de presentar Ag a los linfocitos T. Esta mayor capacidad presentadora de Ag se debe a un aumento de la expresión de las moléculas de superficie co-estimulatorias y a citoquinas como IL12. En presencia de IL12 y las señales co-estimulatorias óptimas, los linfocitos T humanos se diferencian a TH1, mientras que bajo condiciones sub-óptimas se genera IL4. La producción precoz de IFN- $\gamma$ juega un rol central en la capacidad de los RN para controlar la infección por BCG y permite que la administración de la vacuna sea segura. Como además la vacuna BCG induce células de memoria TH1, la recomendación de la Organización Mundial de la Salud (OMS) es vacunar lo más pronto posible después del nacimiento ${ }^{1}$, en los países con alta tasa de TBC; aun cuando no ha sido capaz de prevenir el aumento de la morbilidad y mortalidad por TBC, y su eficacia protectora varíe desde $0 \%$ en USA y el sur de India a $80 \%$ en Reino Unido ${ }^{2}$; ya que se ha demostrado que previene $64-78 \%$ de los casos de meningitis TBC y TBC miliar en niños ${ }^{3-7}$. La vacuna BCG oral fue introducida en Chile en 1930, y en 1938 se inició la vacunación con BCG inyectable con controles previos y posteriores de prueba de tuberculina, constituyéndose sólo en la década del 50, en forma definitiva, como recurso de inmunización ${ }^{8}$. La OMS la incorporó en los programas de inmunización en 19749. En la mayoría de los niños la inoculación de la vacuna BCG es segura, pero puede asociarse a efectos adversos tales como lesiones en el sitio de inoculación, adenitis y, en contados casos, a enfermedades sistémicas. Estos podrían variar con el tipo de cepa, las propiedades físico-químicas de la vacuna y la carga bacilar ${ }^{9,10}$, pero especialmente de los factores propios del hospedero. Las tasas reportadas de adenitis BCG varían ampliamente en un rango de 0 a $17 \%{ }^{11}$. La incidencia de enfermedad BCG diseminada notificada es 0,19 a 1,56 casos por millón de niños vacunados ${ }^{12-15}$.

Los casos de diseminación BCG han sido reportados desde $1951^{15}$. Un estudio retrospectivo nacional francés entre 1974-1994 encontró una prevalencia de 0,59 casos por millón de niños vacunados nacidos en Francia ${ }^{16}$. En 1989 se reportó los primeros nueve casos chilenos de infección por BCG en niños con inmunodeficiencias primarias (IDP), uno de los cuales es de Puerto Montt, observados en un período de 10 años (1976-1986), lo que representa un riesgo de 3,4 por 1 millón de $\mathrm{RN}$ vacunados ${ }^{17}$. En el año 2010 se reportaron cinco casos en la Región de Los Lagos, no asociados a IDP factibles de diagnosticar con estudio inmunológico habitual -tres de evolución letal- en los cuales no fue posible identificar el defecto inmunitario subyacente, y dos de buena evolución en los que se pudo hacer el estudio completo posteriormente, los que están incluidos en el presente reporte $^{18}$. Un estudio retrospectivo nacional chileno será reportado próximamente.
La infección diseminada por BCG es una complicación inhabitual de la vacunación, que ocurre en pacientes con inmunidad alterada ${ }^{19-22}$. Puede haber un riesgo mayor de complicaciones por vacuna BCG en niños infectados por $\mathrm{VIH}$, pero el riesgo no sobrepasa los beneficios de vacunación $\mathrm{BCG}$ en poblaciones con alto riesgo de contraer la TBC durante la infancia y niñez ${ }^{3,4}$. Las tasas de mortalidad por enfermedad BCG notificadas oscilan entre 50 y $70 \%{ }^{23,24}$.

A diferencia con los pacientes que tienen alguna de las IDP de mayor frecuencia, que afectan las respuestas inmune innata y adaptativa en sus componentes humoral o celular (actualmente más de 150 tipos) determinadas genéticamente $^{25}$, y son susceptibles a infecciones recurrentes o graves con microorganismos de baja o alta virulencia, tanto virales, como bacterianas, protozoarias y micóticas, algunos enfermos padecen principalmente de infecciones por bacterias intracelulares de los géneros Mycobacterium y Salmonella que ocurre hasta en 50\% de los pacientes y en algunos casos se ha notificado infecciones por citomegalovirus, Listeria monocytogenes, herpes simplex e Histoplasma $\mathrm{spp}^{21,26-28}$. Ellos forman parte del SMEM. Además de la enfermedad por micobacterias poco virulentas, en este síndrome se ha reportado mayor frecuencia de infecciones por Candida $\mathrm{spp}^{29}$, situación observada en nuestros pacientes, uno de los cuales forma parte de la referencia 29. Por tanto, luego de descartar la presencia de IDP de mayor ocurrencia e ID adquirida, con los exámenes inmunológicos básicos de rutina, es necesario estudiar otros defectos de la respuesta inmune menos frecuentes, como los incluidos en el grupo de defectos congénitos de los fagocitos en número, función o ambos, entre los cuales está SMEM, el que incluye un subtipo (OMIM 601406) $)^{25,29}$ materia de este reporte, quedando aún un grupo de pacientes que tienen enfermedad BCG de etiología aún no precisada, en los cuales es posible que existan otros defectos aún no conocidos ${ }^{21,30,31}$.

Dado que el estudio inmunológico de rutina de estos pacientes es normal, el diagnóstico requiere de investigación específica del circuito IL-12-IFN- $\gamma^{28,30,31}$ combinado o no con el secuenciamiento del exoma. En las formas diseminadas, las características clínicas se correlacionan con el tipo de lesión histopatológica, de tal modo que los niños con granuloma BCG tipo lepromatoso generalmente mueren de la infección, mientras que los niños con granulomas tuberculoídeos tienen un pronóstico favorable ${ }^{32,33}$.

Nosotros reportamos dos lactantes con enfermedad secundaria a la vacuna BCG administrada al nacer, los que presentaron adenomegalia axilar izquierda recurrente antes de los cuatro meses de edad, diagnosticados con posterioridad a otros cinco casos que consultaron también en el Hospital de Puerto Montt y que fueron motivo de una publicación anterior (tres de evolución letal y dos que sobreviven aún); llama la atención que los dos últimos pa- 
cientes de esa publicación que coincidentemente tuvieron buena evolución, son primas hermanas en segundo grado entre sí, y son al mismo tiempo primas hermanas en $1^{\circ}$ y $2^{\circ}$ grado respectivamente con el caso que detallamos en esta notificación en primer lugar y que señalamos como número 3, para demostrar la ocurrencia de tres casos dentro de una misma familia (Figura 2). El caso 4 en el que no se pudo demostrar relación familiar con los anteriores y por tanto constituye un caso aislado, presentó además candidiasis oral extensa o recurrente al diagnóstico y durante el tratamiento. En todos los pacientes, los exámenes bacteriológicos e histopatológicos confirmaron la etiología BCG, y concordando con la buena evolución clínica presentada correspondieron al tipo histológico tuberculoídeo diferenciado.

Como el estudio inmunológico disponible en Chile fue normal (Tabla 2) descartando por tanto IDP comunes e ID secundarias incluyendo VIH, se envió muestras al centro de referencia internacional previamente señalado para los exámenes del eje IL-12-IFN- $\gamma$.

Se ha descrito que alrededor de la mitad de los pacientes con enfermedad BCG en los que no se encontró una IDP frecuente o ID secundaria, pueden tener un defecto heredado del eje IL12-IFN- $\gamma$. Por otra parte, las formas familiares y consanguinidad parental en estos enfermos son frecuentes lo que apoya el origen genético. En aquellos en los que se ha demostrado una alteración en el circuito IL12-IFN- $\gamma$, se ha detectado herencia autosómica recesiva en la mayoría de los casos, autosómica dominante y recesiva ligada a $\mathrm{X}^{34}$.

De nuestros casos destaca la relación de parentesco entre los tres primeros casos (Figura 2), confirmándose el mismo defecto genético en los casos 1 y 3 , en que ambos padres y ambas madres son hermanas, en tanto en la paciente 2 , pese a la relación de parentesco con las dos primeras (hija de un primo hermano de los padres de las pacientes 1 y 3 ), no se ha logrado identificar a la fecha el defecto genético que explique la presencia de la enfermedad BCG; sin embargo, continúa en estudio en el centro genético mencionado anteriormente.

El caso 4 no tiene antecedente conocido de consanguinidad o relación familiar con los otros casos; sin embargo, la cercanía geográfica hace presumir que podría haber algún familiar en común en generaciones anteriores y es posible que a futuro pueda demostrarse la presencia de un gen fundador semejante al encontrado en los pacientes argentinos ${ }^{35}$-no se ha estudiado en nuestros pacientes-, puesto que comparten hechos históricos como la colonización española inicial y las posteriores migraciones de otros países europeos, en especial en el sur de Chile.

Estas alteraciones se manifiestan generalmente en la niñez, aunque también pueden aparecer durante la adultez. La gravedad del fenotipo clínico depende del genotipo.
Generalmente los pacientes con deficiencias de IL12, IL-12p40, y sus receptores tienen síntomas leves, con formación de un granuloma diferenciado en respuesta a la vacunación $\mathrm{BCG}$, aunque retardado. El fenotipo clínico de los pacientes con deficiencia parcial de IFN- $\gamma$ R puede ser tan leve como en la deficiencia de receptores de IL12, presentando infecciones que pueden ser curables a distintas edades y si se requiere suplementar con IFN- $\gamma$ probablemente el resultado será efectivo.

Por otra parte, los pacientes con deficiencias completas de IFN- $\gamma$ R 1 o R2 padecen una forma grave del síndrome, con infección BCG post vacuna no controlada, devastadora, e histológicamente es posible observar lesiones tipo lepromatoso; pueden responder a antimicrobianos pero recaen cuando éstos se discontinúan.

Así, la discriminación rápida entre deficiencia completa de receptores de IFN- $\gamma$ y los otros defectos es un paso importante para planificar el manejo y orientar el pronóstico ${ }^{36}$. Las mutaciones del gen IL-12R $\beta 1$ represen$\tan 40 \%$ entre 220 pacientes con SMEM de 40 países en cinco continentes, en los que se ha determinado el defecto genético desde 1998 y hasta la fecha se han determinado más de 40 mutaciones distintas ${ }^{19,20,33-37}$.

La identificación de la deficiencia del receptor $\beta 1$ de IL-12 en tres de nuestros cuatro pacientes se logró con el envío de muestras de todos los pacientes y los familiares directos al Laboratorio de Genética Humana de Enfermedades Infecciosas de la Facultad de Medicina Necker, Institut Imagine-París, Francia.

Hay pocas guías de diagnóstico y tratamiento para enfermedad BCG; más aún, el test de susceptibilidad a fármacos anti-TBC no se realiza rutinariamente a las micobacterias aisladas especialmente en países con recursos $\operatorname{limitados}^{38}$. En Chile, el estudio de sensibilidad para BCG no se realiza en los laboratorios clínicos que identifican rutinariamente por cultivo las micobacterias como complejo M. tuberculosis, que incluye M. tuberculosis, M. bovis, M. bovis BCG y otras especies ${ }^{39}$. Los métodos basados en reacción de polimerasa en cadena (RPC) disponibles en el Instituto de Salud Pública de Chile, permiten la rápida y segura diferenciación del complejo M. tuberculosis y el desarrollo de técnicas para identificar resistencia a fármacos ${ }^{39}$. Se sabe que el BCG tiene resistencia intrínseca a pirazinamida y se ha descrito enfermedad BCG con resistencia a múltiples fármacos, con resultado fatal $\mathrm{o}$ crónico $^{40}$. Para el manejo de pacientes con sospecha de enfermedad $\mathrm{BCG}$ es esencial asegurar la rápida y segura confirmación de $M$. bovis BCG, el uso de dosis más altas de antimicrobianos específicos y por tiempo prolongado ${ }^{40}$. En nuestro país no hay recomendaciones específicas de tratamiento para la enfermedad BCG, por lo cual los casos 1 y 2 fueron tratados con esquema antiTBC vigente, aunque por períodos más prolongados, de acuerdo a la respuesta clínica, e incluyeron el uso de pirazinamida. 
Los casos nuevos que incluye este reporte, utilizaron el esquema recomendado por Hesseling y cols., para pacientes no portadores de $\mathrm{VIH}$ con infección diseminada por $\mathrm{BCG}^{23}$. A todos se les efectuó drenaje, extirpación y biopsia de los nódulos axilares, incluso en forma repetida, ya que se ha descrito que la propensión a desarrollar resistencia posterior apoya la resección quirúrgica precoz y la escisión de nódulos axilares persistentes para eliminar un foco crónico de infección ${ }^{41}$. Todos tuvieron una respuesta clínica lenta, pero satisfactoria y a la fecha se encuentran clínicamente sin enfermedad BCG, aunque el caso 4 presenta un cuadro dermatológico de etiología poco clara, sin elementos histológicos de vasculitis leucocitoclástica cutánea, enfermedad que ha sido asociada a Salmonella Enteritidis, reportada previamente en pacientes con deficiencia del receptor $\beta-1$ de IL-12 ${ }^{42-44}$.

Otras alternativas terapéuticas como el uso de interferón $\gamma^{45-47}$ no fueron consideradas, dada la evolución favorable con los esquemas de tratamiento usados.

Este reporte debiera estimular la sospecha diagnóstica de defectos heredados del eje IL12-IFN- $\gamma$ en los pacientes pediátricos y adultos con reacciones adversas al uso de la vacuna BCG considerando que el retardo en el diagnóstico, los esquemas de tratamiento inapropiados, en cuanto a fármacos anti-TBC y dosis usadas, la mala adherencia y la resistencia podrían influir en la respuesta al tratamiento.

Agradecimientos: Los autores agradecen a Jean Laurent Casanova y Jacinta Bustamante por el permanente apoyo y motivación para la publicación de estos casos, y por el estudio del eje IL-12/IFN- $\gamma$ realizado, y a Marcela Amtmann por la excelente colaboración bibliográfica y preparación del manuscrito.

\section{Resumen}

La enfermedad por el bacilo de Calmette-Guérin (BCG) ha sido reportada en relación a inmunodeficiencias primarias, secundarias y en el síndrome clínico denominado susceptibilidad mendeliana a enfermedades micobacterianas. La investigación de este síndrome ha llevado a la identificación de defectos en el eje interleuquina (IL)- 12/ interferón gamma (IL-12/IFN- $\gamma$ ), habiéndose identificado hasta hoy mutaciones en siete genes autosómicos y dos ligados al cromosoma X. En estos pacientes, las infecciones localizadas o generalizadas por BCG vacunal son comunes. Reportamos una serie clínica constituida por dos lactantes con adenomegalia axilar izquierda recurrente secundaria a vacunación BCG al nacer; uno de ellos integrante de una familia con dos casos reportados previamente y un caso aislado, diagnosticados consecutivamente en Puerto Montt, Chile, con el objetivo de notificar los primeros casos chilenos de diseminación BCG en niños sin inmunodeficiencia previa conocida, en los que se logró identificar la deficiencia inmune subyacente pese a no disponer en el país del estudio específico del eje (IL-12/IFN- $\gamma$ ). La sospecha diagnóstica y colaboración internacional permitieron identificar en dos de los tres casos familiares y en el caso aislado, la deficiencia del receptor $\beta 1$ de IL 12 (IL12R $\beta 1$ ).

\section{Referencias bibliográficas}

1.- Marchant A, Goetghebuer T, Ota M O, Wolfe I, Ceesay S J, De Groote D, et al. Newborns develop a Th1-type immune response to Mycobacterium bovis bacillus CalmetteGuérin vaccination. J Immunol 1999; 163 : 2249-55.

2.- Girard M P, Fruth U and Kieny M P. A review of vaccine research and development: tuberculosis. Vaccine 2005; 23: 5725-31.

3.- O’Brien K L, Ruff A J, Louis M A, Desormeaux J, Joseph D J, McBrien M, et al. Bacillus Calmette-Guérin complications in children born to HIV-1-infected women with a review of the literature. Pediatrics 1995; 95 : 414-8.

4.- Hesseling A C, Marais B J, Gie R P, Schaaf H S, Fine P E, Godfrey-Faussett P, et al. The risk of disseminated Bacille CalmetteGuerin (BCG) disease in HIV-infected children. Vaccine 2007; 25: 14-8.

5.- Sutherland I. An assessment of the value of BCG vaccination in England and Wales. Pneumologie 1990; 44: 653-4.
6.- Colditz G A, Berkey C S, Mosteller F, Brewer T F, Wilson M E, Burdick E, et al. The efficacy of bacillus Calmette-Guérin vaccination of newborns and infants in the prevention of tuberculosis: meta-analyses of the published literature. Pediatrics 1995; 96: 29-35.

7.- Colditz G A, Brewer T F, Berkey C S, Wilson M E, Burdick E, Fineberg H V, et al. Efficacy of BCG vaccine in the prevention of tuberculosis. Meta-analysis of the published literature. JAMA 1994;271: 698-702.

8.- Laval E. Sobre la transmisión de la tuberculosis y los primeros ensayos del BCG. Rev Chilena Infectol 2003; 20: 51-3.

9.- Hesseling A C, Schaaf H S, Hanekom W A, Beyers N, Cotton M F, Gie R P, et al. Danish bacille Calmette-Guérin vaccine-induced disease in human immunodeficiency virusinfected children. Clin Infect Dis 2003; 37: 1226-33.

10.- Fine P E M, Carneiro I A M, Milstein J B, Clements $\mathrm{C} J$. Issues relating to the use of BCG immunization programmes-a discussion document. World Health Organization (WHO) document no. WHO/V\&B/99.23. Geneva:
WHO, 1999.

11.- Milstein J B, Gibson J J. Quality control of BCG by WHO: a review of factors that may influence vaccine effectiveness and safety. Bull World Health Org 1990; 68: 93-108.

12.- Nicol M, Eley B, Kibel M, Hussey G. Intradermal $\mathrm{BCG}$ vaccination--adverse reactions and their management. S Afr Med J 2002; 92 : $39-42$.

13.- Jouanguy E, Lamhamedi-Cherradi S, Lammas D, Dorman S E, Fondanèche M C, Dupuis S, et al. A human IFNGR1 small deletion hotspot associated with dominant susceptibility to mycobacterial infection. Nat Genet 1999; 21: 370-8.

14.- Lotte A, Wasz-Hockert O, Poisson N, Engbaek H, Landmann H, Quast U, et al. Second IUATLD study on complications induced by intradermal BCG-vaccination. Bull Int Union Tuberc Lung Dis 1988; 63: 47-59.

15.- Casanova J L, Jouanguy E, Lamhamedi S, Blanche S, Fischer A. Immunological conditions of children with BCG disseminated infection. Lancet. 1995; 346: 581.

16.- Casanova J L, Blanche S, Emile J F, 
Jouanguy E, Lamhamedi S, Altare F, et al. Idiopathic disseminated bacillus CalmetteGuérin infection: a French national retrospective study. Pediatrics 1996; 98: 774-8.

17.- González B, Moreno S, Burdach R, Valenzuela M T, Henríquez A, Ramos M I. Clinical presentation of Bacillus CalmetteGuérin infections in patients with immunodeficiency syndromes. Pediatr Infect Dis J 1989; 8: 201-6.

18.- Strickler A, González B, Boza M L, González B, Márquez G, Bustamante J. Infección diseminada por BCG en la Región de Los Lagos, Chile: Reporte de cinco casos clínicos. Rev Chil Enferm Respir 2009; 25: 29-38.

19.- Talbot E A, Perkins M D, Silva S F, Frothingham R. Disseminated bacille Calmette-Guerin disease after vaccination: case report and review. Clin Infect Dis 1997; 24: 1139-46.

20.- Bernatowska E A, Wolska-Kusnierz B, Pac M, Kurenko-Deptuch M, Zwolska Z, Casanova J L, et al. Disseminated bacillus Calmette-Guérin infection and immunodeficiency. Emerg Infect Dis 2007; 13: 799-801.

21.- Norouzi S, Aghamohammadi A, Mamishi S, Rosenzweig S D, Rezaei N. Bacillus CalmetteGuérin (BCG) complications associated with primary immunodeficiency diseases. J Infect 2012; 64: 543-54.

22.- Bernatowska E A, Wolska-Kusnierz B, Pac M, Kurenko-Deptuch M, Pietrucha B, Zwolska Z, et al. Risk of BCG infection in primary immunodeficiency children. Proposal of diagnostic, prophylactic and therapeutic guidelines for disseminated BCG based on experience in the Department of Immunology, Children's Memorial Health Institute in Warsaw between 1980-2006. Centr Eur J Immunol 2007; 32: 221-5.

23.- Hesseling A C, Rabie H, Marais B J, Manders M, Lips M, Schaaf H S, et al. Bacille Calmette-Guérin vaccine-induced disease in HIV-infected and HIV-uninfected children. Clin Infect Dis 2006; 42: 548-58.

24.- Nicol M, Eley B, Kibel M, Hussey G. Intradermal BCG vaccination--adverse reactions and their management. S Afr Med J 2002; 92: 39-42.

25.- Al-Herz W, Bousfiha A, Casanova J L, Chapel $\mathrm{H}$, Conley M E, Cunningham-Rundles $\mathrm{Ch}$, et al. Primary immunodeficiency diseases: an update on the classification from the International Union of Immunological Societies Expert Committee for Primary Immunodeficiency. Frontiers in Immunology 2011 (2); 54: 1-26.

26.- Remus N, Reichenbach J, Picard C, Rietschel C, Wood P, Lammas D, et al. Impaired interferon gamma-mediated immunity and susceptibility to mycobacterial infection in childhood. Pediatr
Res 2001; 50: 8-13.

27.- Filipe-Santos O, Bustamante J, Chapgier A, Vogt G, de Beaucoudrey L, Feinberg J, et al. Inborn errors of IL-12/23- and IFN-gammamediated immunity: molecular, cellular, and clinical features. Semin Immunol 2006; 18: 347-61

28.- Pedraza S, Aldana R, Herrera M T, Torres M, Bustamante J. Infecciones por Mycobacterium y Salmonella en pacientes con inmunodeficiencias asociadas al circuito IL-12/IL-23-IFN- $\gamma$ : aspectos biológicos y clínicos. Enf Inf Microbiol 2007; 23: 88-99.

29.- Ouederni M, Sanal O, Ikinciogullari A, Tezcan I, Dogu F, Sologuren I. Clinical features of candidiasis in patients with inherited interleukin-12 receptor $\beta 1$ deficiency. Clin Infect Dis 2014; 58 (2): 204-13.

30.- Prando C, Samarina A, Bustamante J, BoissonDupuis S, Cobat A, Picard C, et al. Inherited IL-12p40 deficiency: genetic, immunologic, and clinical features of 49 patients from 30 kindreds. Medicine (Baltimore) 2013; 92: 109-22.

31.- Talbot E A, Perkins M D, Silva S F, Frothingham R. Disseminated bacille Calmette-Guérin disease after vaccination: case report and review. Clin Infect Dis. 1997; 24: 1139-46.

32.- Mansouri D, Adimi P, Mirsaeidi M, Mansouri N, Khalilzadeh S, Masjedi M R, et al. Inherited disorders of the IL-12-IFNgamma axis in patients with disseminated BCG infection. Eur J Pediatr 2005; 164: 753-7.

33.- Abramowsky C, González B, Sorensen R U. Disseminated bacillus Calmette-Guérin infections in patients with primary immunodeficiencies. Am J Clin Pathol 1993; 100: $52-6$

34.- Emile J F, Patey N, Altare F, Lamhamedi S, Jouanguy E, Boman F, et al. Correlation of granuloma structure with clinical outcome defines two types of idiopathic disseminated BCG infection. J Pathol. 1997; 181: 25-30.

35.- de Beaucoudrey L, Samarina A, Bustamante J, Cobat A, Boisson-Dupuis S, Feinberg J, et al Revisiting human IL-12R $\beta 1$ deficiency: a survey of 141 patients from 30 countries. Medicine (Baltimore). 2010; 89: 381-402.

36.- Yancoski J, Rocco C, Bernasconi A, Oleastro M, Bezrodnik L, Vrátnica C, et al. A 475 years-old founder effect involving IL12RB1: a highly prevalent mutation conferring Mendelian Susceptibility to Mycobacterial Diseases in European descendants. Infect Genet Evol 2009; 9: 574-80.

37.- Hesseling A C, Schaaf H S, Victor T, Beyers N, Marais B J, Cotton M F, et al. Resistant Mycobacterium bovis Bacillus CalmetteGuérin disease: implications for management of Bacillus Calmette-Guérin Disease in human immunodeficiency virus-infected children. Pediatr Infect Dis J 2004; 23: 476-9.
38.- van de Vosse E L, Haverkamp M H, Ramírez-Alejo N, Martínez-Gallo M, Blancas-Galicia L, Metin A, et al. IL-12R $\beta 1$ deficiency: mutation update and description of the IL12RB1 variation database. Hum Mutat 2013; 34: 1329-39.

39.- No authors listed. Diagnostic Standards and Classification of Tuberculosis in Adults and Children. This official statement of the American Thoracic Society and the Centers for Disease Control and Prevention was adopted by the ATS Board of Directors, July 1999. This statement was endorsed by the Council of the Infectious Disease Society of America, September 1999. Am J Respir Crit Care Med 2000; 161: 1376-95.

40.- Su W J. Recent advances in the molecular diagnosis of tuberculosis. J Microbiol Immunol Infect. 2002; 35: 209-14.

41.- Rosenzweig S D, Yancoski J, Bernasconi A, Krasovec S, Marciano B E, Casimir L, et al. Thirteen years of culture-positive $M$. bovis-BCG infection in an IL-12R beta1 deficient patient: treatment and outcome. J Infect 2006; 52: e69-72.

42.- Hengster P, Sölder B, Fille M, Menardi G. Surgical treatment of bacillus Calmette Guérin lymphadenitis. World J Surg. 1997; 21: 520-3.

43.- Savino F, Lupica M M, Tarasco V, Locatelli E, Viola S, Cordero di Montezemolo L, et al. Acute hemorrhagic edema of infancy: a troubling cutaneous presentation with a self-limiting course. Pediatr Dermatol. 2013; 30: e149-52.

44.- Kutukculer N, Genel F, Aksu G, Karapinar B, Ozturk C, Cavusoglu C, et al. Cutaneous leukocytoclastic vasculitis in a child with interleukin-12 receptor beta-1 deficiency. $\mathrm{J}$ Pediatr 2006; 148: 407-9.

45.- Sanal O, Turul T, De Boer T, Van de Vosse E, Yalcin I, Tezcan I, et al. Presentation of interleukin-12/-23 receptor beta 1 deficiency with various clinical symptoms of Salmonella infections. J Clin Immunol. 2006; 26: 1-6.

46.- Alangari A A, Al-Zamil F, Al-Mazrou A, Al-Muhsen S, Boisson-Dupuis S, Awadallah S, et al. Treatment of disseminated mycobacterial infection with high-dose IFN- $\gamma$ in a patient with IL-12Rß1 deficiency. Clin Dev Immunol 2011; 2011: 691956 http://dx.doi. org/10.1155/2011/691956.

47.- Ulrichs T, Fieschi C, Nevicka E, Hahn H, Brezina M, Kaufmann S H, et al. Variable outcome of experimental interferon-gamma therapy of disseminated Bacillus CalmetteGuerin infection in two unrelated interleukin12R beta1-deficient Slovakian children. Eur J Pediatr 2005; 164: 166-72.

48.- Palamaro L, Giardino G, Santamaría F, Romano R, Fusco A, Montella S, et al. Interleukin 12 receptor deficiency in a child with recurrent bronchopneumonia and very high IgE levels. Ital J Pediatr 2012; 38: 46. 